\title{
A 310 nm Optically Pumped AIGaN Vertical-Cavity Surface-Emitting Laser
}

Filip Hjort,* Johannes Enslin, Munise Cobet, Michael A. Bergmann, Johan Gustavsson, Tim Kolbe, Arne Knauer, Felix Nippert, Ines Häusler, Markus R. Wagner, Tim Wernicke, Michael Kneissl, and Åsa Haglund

Cite This: ACS Photonics 2021, 8, 135-141

Read Online

ACCESS | 네 Metrics \& More | 回 Article Recommendations | sl Supporting Information

ABSTRACT: Ultraviolet light is essential for disinfection, fluorescence excitation, curing, and medical treatment. An ultraviolet light source with the small footprint and excellent optical characteristics of vertical-cavity surfaceemitting lasers (VCSELs) may enable new applications in all these areas. Until now, there have only been a few demonstrations of ultraviolet-emitting VCSELs, mainly optically pumped, and all with low Al-content AlGaN cavities and emission near the bandgap of $\mathrm{GaN}(360 \mathrm{~nm})$. Here, we demonstrate an optically pumped VCSEL emitting in the UVB spectrum $(280-320 \mathrm{~nm})$ at room temperature, having an $\mathrm{Al}_{0.60} \mathrm{Ga}_{0.40} \mathrm{~N}$ cavity between two dielectric distributed Bragg reflectors. The double dielectric distributed Bragg reflector design was realized by substrate removal using electrochemical etching. Our method is further extendable to even shorter wavelengths, which would establish a technology that enables VCSEL emission from UVA $(320-400 \mathrm{~nm})$ to UVC $(<280 \mathrm{~nm})$.

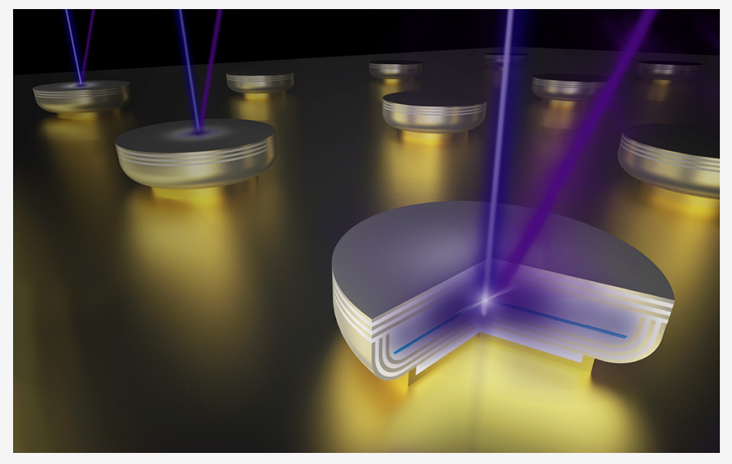

KEYWORDS: vertical-cavity surface-emitting laser, AlGaN, ultraviolet, UVB, electrochemical etching, dielectric DBR

V ertical-cavity surface-emitting lasers (VCSELs) have circular-symmetric beams, low threshold currents, 2Darray manufacturability, and a compatibility with on-wafer testing, leading to low production costs. ${ }^{1}$ Due to these advantages, infrared-emitting GaAs-based VCSELs constitute a rapidly growing billion-dollar industry and are, for example, used in data communication, sensing, and illumination. In addition, infrared VCSEL arrays delivering power densities over $100 \mathrm{~W} / \mathrm{cm}^{2}$ are used for industrial heating. ${ }^{2}$ The VCSEL market will be further expanded when visible-emitting GaNbased VCSELs are commercialized. This will soon be a reality based on the recent immense improvement in performance ${ }^{3-8}$ enabled by advances in thermal management, ${ }^{3,6,9}$ optical confinement, ${ }^{5,10,11}$ mirror reflectivity,,$^{8,18,12}$ and electrical injection.,13 Part of these advances may also boost the development of ultraviolet (UV) AlGaN-based VCSELs, sources with a higher brightness than LEDs. UV light is used for water and surface disinfection, fluorescence excitation, curing, and medical treatment, ${ }^{14}$ and the realization of UV VCSELs could, for example, enable energy-efficient, highthroughput, and compact water purification systems based upon 2D-laser arrays. In addition, new applications in medical diagnostics and therapy, atomic clocks, and UV curing could be facilitated. However, previously reported UV VCSELs were mainly optically pumped and all emitted in the UVA (320$400 \mathrm{~nm}) .{ }^{15-21}$ The low Al-content AlGaN cavities employed in the previous demonstrations are limited to emission wavelengths near the bandgap of $\mathrm{GaN}(360 \mathrm{~nm})$ and prevent extension into the UVB $(280-320 \mathrm{~nm})$ and UVC $(<280 \mathrm{~nm})$ spectral ranges.

The limited progress in UV VCSELs so far is caused by challenges in extending the electrical injection schemes and mirror solutions of GaN-based VCSELs to AlGaN-based devices. Due to the low lateral conductivity of $\mathrm{p}-\mathrm{GaN}$, most blue-emitting VCSELs use indium tin oxide (ITO) as intracavity p-contact and current spreader, ${ }^{3-6,8-10,22}$ but ITO is not suitable in the UV due to its high optical absorption. ${ }^{13}$ Alternatively, tunnel junctions can be used, enabling current spreading by a highly conductive $n$-doped layer on top. To be suitable for VCSELs, a tunnel junction needs to have low resistivity, be stable at high current densities, and have very low optical absorption, all of which is especially challenging to achieve for UV devices. Nevertheless, recent advances in

Received: September 4, 2020

Published: December 17, 2020 


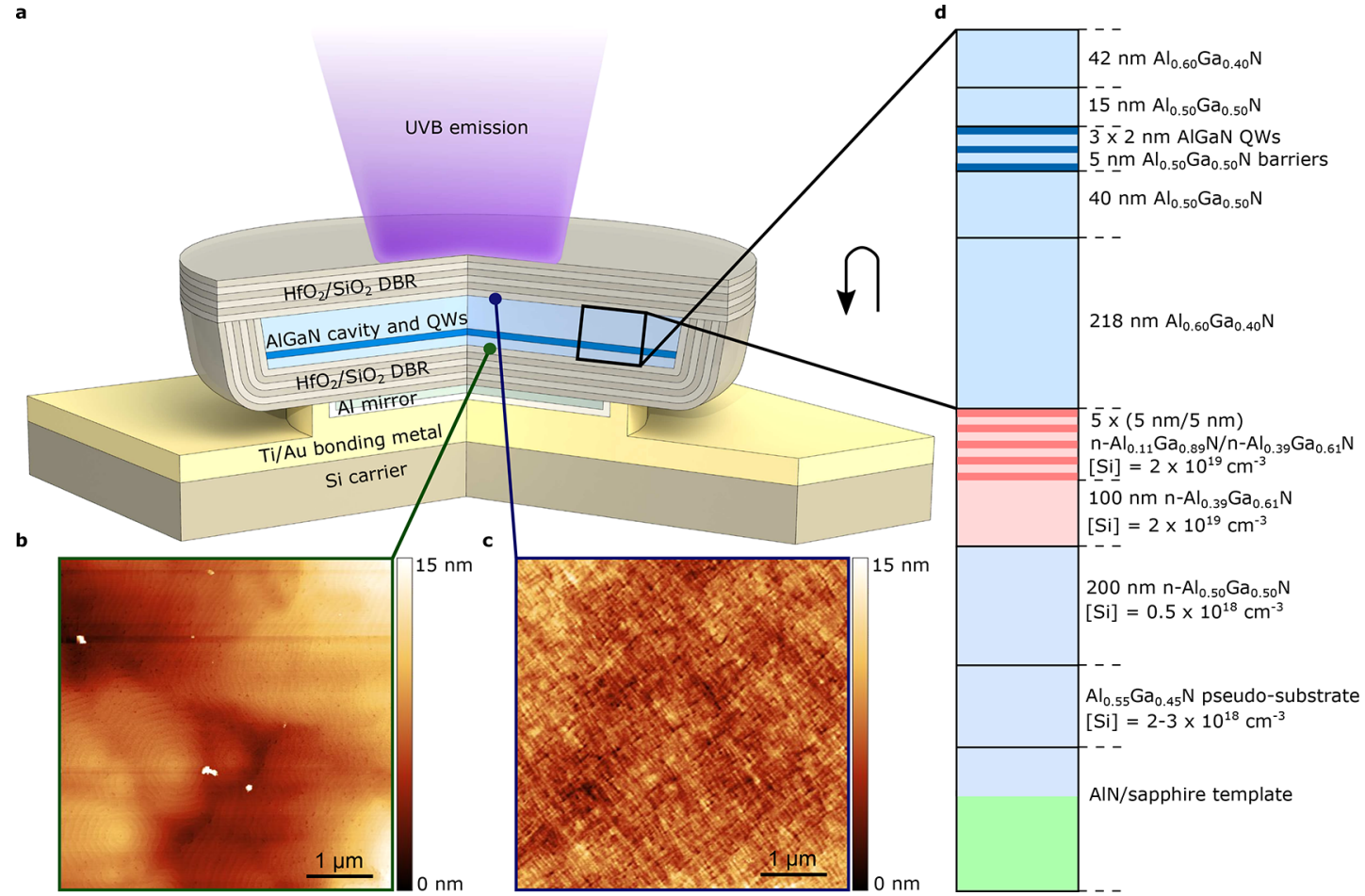

Figure 1. UVB VCSEL structure, surface topography, and epitaxial structure. (a) Schematic structure of the UVB VCSEL. (b) Atomic force microscopy images of the as-grown metal-polar $\mathrm{Al}_{0.6} \mathrm{Ga}_{0.4} \mathrm{~N}$ surface and (c) of the N-polar surface exposed by the electrochemical etching of the sacrificial layer. (d) As-grown epitaxial layer structure. The multilayered sacrificial layer is marked in red.
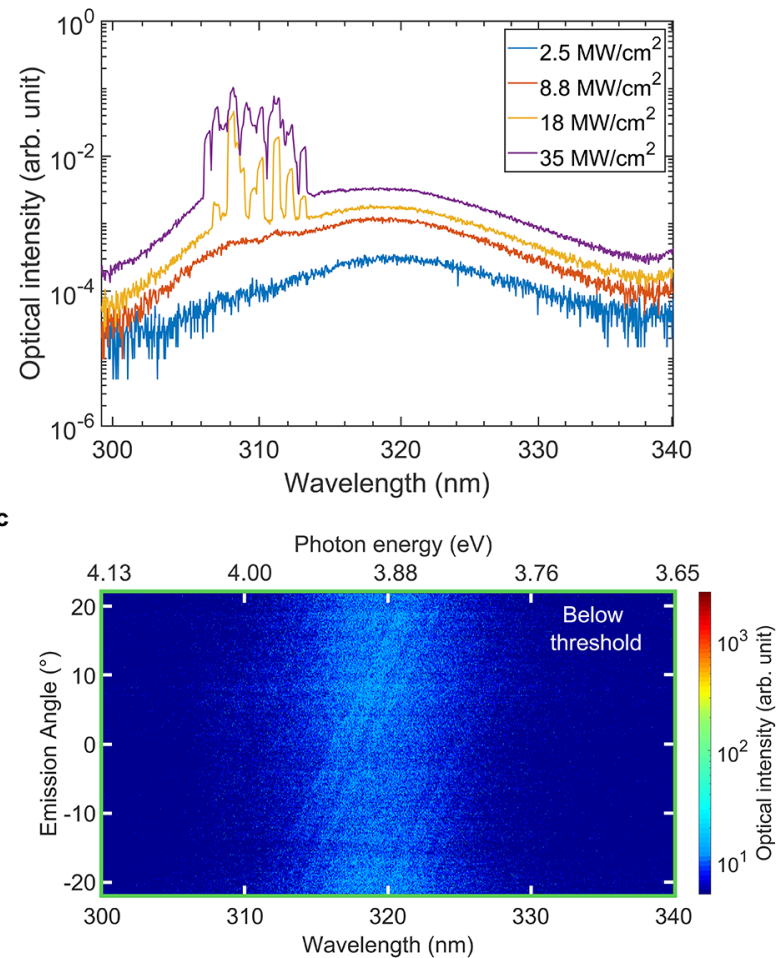
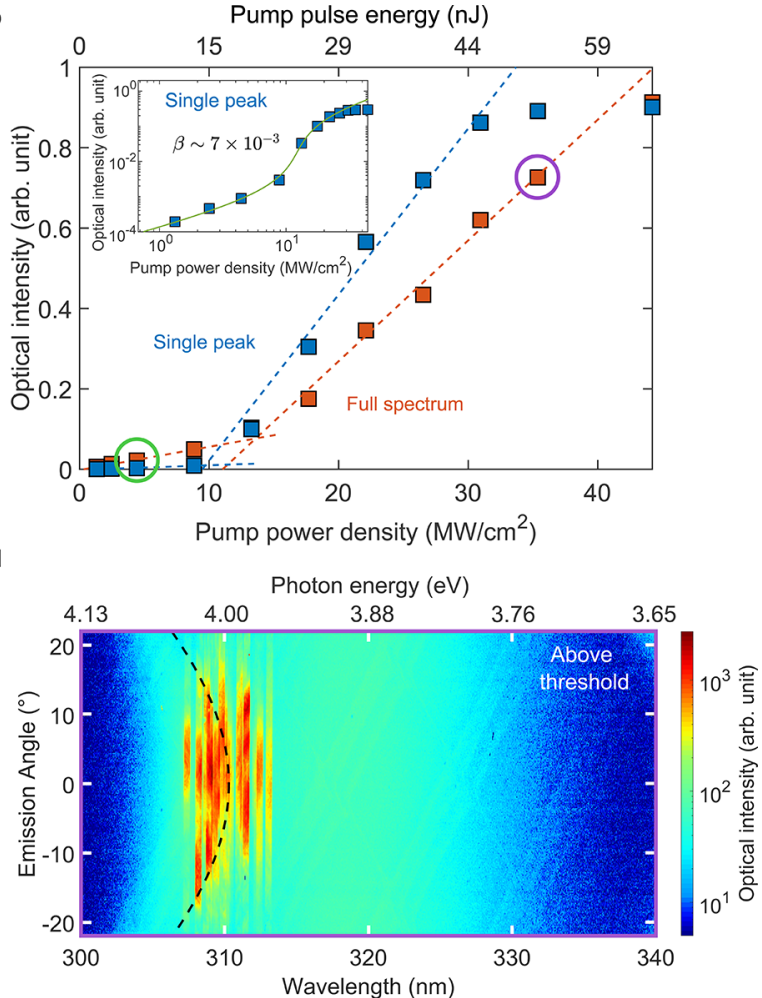

Figure 2. UVB VCSEL output intensity and spectral characteristics. (a) Logarithmic-scale photoluminescence (PL) emission spectra at room temperature for different pump power densities under pulsed optical pumping. (b) Optical emission intensity at room temperature, integrated over the entire spectrum and around a single lasing peak, as a function of pump power density and energy per pulse. The inset shows the single peak emission plotted versus pump energy in log-log-scale, where the green line is a fit to the measured data. (c) Logarithmic-scale, angle-resolved spectra of the emission below threshold and (d) above threshold. The green and purple circles in (b) mark the data points corresponding to (c) and (d), and the black dashed line in (d) marks the simulated longitudinal cavity mode. The spectral resolution of the measurements is 0.5 nm. 

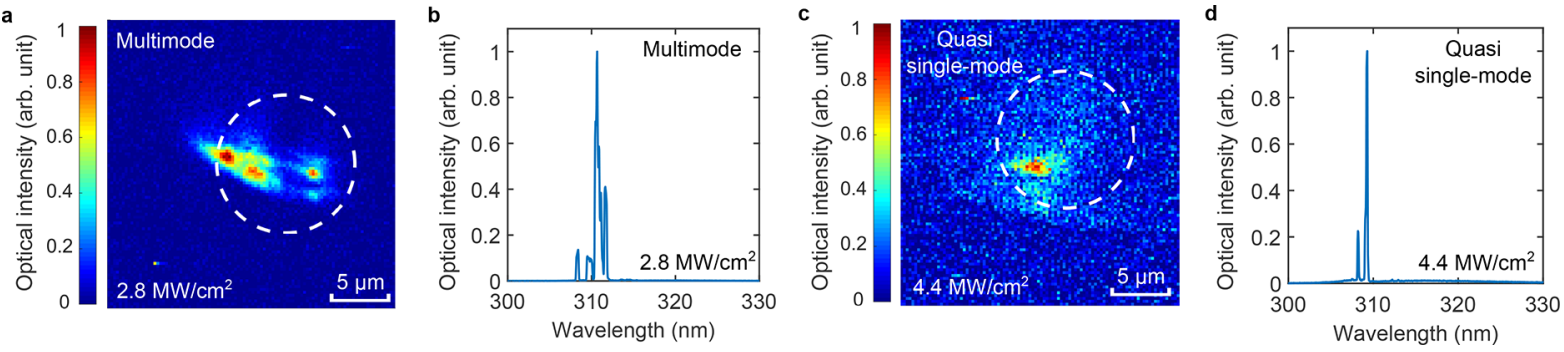

Figure 3. Imaging of UVB VCSEL spatial emission distribution. (a) Spatial emission distribution at the sample surface and (b) spectrum for a multimode device. (c) Spatial emission distribution at the sample surface and (d) spectrum for a quasi single-mode device. The dashed white circles indicate the position of the pump spot. The spectral resolution of the measurements is $0.15 \mathrm{~nm}$.

AlGaN-based tunnel junctions ${ }^{23-25}$ demonstrate their potential as a viable solution for electrical injection of UV VCSELs.

In addition to electrical injection, a remaining challenge is the formation of distributed Bragg reflectors (DBRs) with higher than $99 \%$ reflectivity. Substantial effort has been made to develop epitaxial III-nitride DBRs for UV wavelengths, ${ }^{26-28}$ but a need for low absorption and small in-plane lattice mismatch limits the available $\mathrm{AlGaN}$-composition range, which in turn decreases the maximum refractive index contrast between the individual DBR layers. A small index contrast results in a narrow stopband and makes it difficult to reach the required reflectivity. The performance of III-nitride UVB and UVC DBRs has therefore been comparatively poor, with no demonstration of reflectivities above 99\%. An alternative solution to realize UV VCSELs is to use only dielectric DBRs. In this case, high reflectivity and wide stopbands are more easily achievable, but the substrate must typically be removed to enable deposition of the second dielectric DBR. Furthermore, the exposed surface must be smooth to avoid scattering losses. Unfortunately, for AlGaN-based devices, substrate removal by laser-induced lift-off can lead to cracking of epitaxial layers and to rough surfaces with root-mean-square roughness of $20 \mathrm{~nm}$ or higher, while chemical-mechanical polishing offers poor thickness control. ${ }^{29-31}$ An alternative way to remove the substrate is to use $\mathrm{Al}$-content- and dopingselective electrochemical etching of $\mathrm{AlGaN}$, which has recently been demonstrated by our groups. ${ }^{32,33}$ This method allows for the fabrication of AlGaN membranes with high $\mathrm{Al}$ content, smooth surfaces, and well-defined thicknesses. Owing to these properties, the membranes can be used for a wide variety of thin-film devices. Here, we use this technique to realize a VCSEL emitting at $310 \mathrm{~nm}$ at room temperature under pulsed optical pumping, thereby extending the wavelength range of VCSEL emission further into the UV than previously demonstrated.

The VCSEL is shown schematically in Figure 1a and consists of a $2.5 \lambda \mathrm{Al}_{0.60} \mathrm{Ga}_{0.40} \mathrm{~N}$ cavity including three $\mathrm{AlGaN}$ quantum wells (QWs), sandwiched between two dielectric $\mathrm{HfO}_{2} / \mathrm{SiO}_{2}$ DBRs. The top 10-pair DBR has a stopband centered at $320 \mathrm{~nm}$ and a measured peak reflectivity above $99 \%$. The bottom reflector ends with an additional DBR pair and an $\mathrm{Al}$ mirror. By using an $\mathrm{Al}$ mirror, the reflectivity of the $\mathrm{DBR}$ is enhanced at the emission wavelength. ${ }^{34}$ Even more importantly, the reflectivity is also strongly enhanced on the shortwavelength side of the DBR stopband at the $266 \mathrm{~nm}$ pump wavelength, which promotes recycling of the nonabsorbed pump beam photons (see Supporting Information Sections 1 and 2). The bottom DBR is deposited on the as-grown epitaxial surface, whereas the top DBR is deposited, after flip- chip bonding, on the $\mathrm{N}$-polar $\mathrm{Al}_{0.60} \mathrm{Ga}_{0.40} \mathrm{~N}$ surface exposed by electrochemical etching. The etched surface (Figure 1b) has a root-mean-square roughness of $1.7 \mathrm{~nm}$ on a $5 \times 5 \mu \mathrm{m}^{2}$ area, which is similar to the $2.2 \mathrm{~nm}$ of the as-grown surface (Figure 1c), demonstrating the capability of the electrochemical etching to create very smooth surfaces. On a larger scale, the electrochemically etched surface has an inverted topography of that of the as-grown surface, similar to what we have reported previously. ${ }^{32}$ The smooth etching was achieved by using a multilayered sacrificial layer, which promotes etching at the sacrificial layer and cavity interface; see Figure $1 \mathrm{~d}$. This design enables incorporation of layers with lower $\mathrm{Al}$ content without degrading the crystal quality of the device layers, and the builtin polarization field yields sheets of high carrier concentrations, both of which enhance the etch selectivity ${ }^{32}$ and result in smoother surfaces.

Figure 2a shows the VCSEL's optical emission spectra at room temperature for different pump power densities, while Figure $2 \mathrm{~b}$ shows emission intensity as a function of the pump power density. The emission intensity is calculated by integrating over the entire multimode spectrum as well as around a single peak. Both curves show a clear lasing threshold around $10 \mathrm{MW} / \mathrm{cm}^{2}$, corresponding to a QW threshold carrier density in the $10^{20} \mathrm{~cm}^{-3}$ range, which is a realistic threshold carrier density for AlGaN lasers (see Supporting Information Section 2). The inset in Figure $2 b$ presents the optical intensity of the single peak versus pump power in double-logarithmic scale, and extraction of the spontaneous emission factor $\beta$ from the spectrally filtered emission gives $\beta \approx 7 \times 10^{-3}$. This is similar to what has been reported for GaN-based VCSELs. ${ }^{35,36}$ However, $\beta$ is generally overestimated, as precise determination requires both spectral and spatial filtering. ${ }^{37}$

In Figure 2c,d, the angle-resolved spectrum of the emission is shown both below and above threshold and reveals a spectral narrowing as well as a clear beam width narrowing around threshold. As can be seen in Figure 2c, QW excitonic emission at $320 \mathrm{~nm}$, which is unfiltered by the cavity and thus nondispersive, is dominating below threshold. In Figure 2d, above threshold, multiple lasing peaks with resolution-limited line widths and narrow angular beam widths (full width at halfmaximum below $15^{\circ}$ ) are visible around $310 \mathrm{~nm}$ at an angulardispersive longitudinal cavity mode. These characteristics support the claim of lasing in the vertical cavity. The deviation in lasing wavelength from the designed $320 \mathrm{~nm}$ is due to a slightly shorter realized optical cavity length compared to targeted (see Supporting Information Section 3). This cavity length deviation results in a high absorption of the cavity mode at low pumping powers and may thus explain why the cavity mode is only visible at higher pump power densities. In 
Supporting Information Figure S3, which shows the angleresolved spectrum of a similar sample, the $2.5 \lambda$-cavity mode is easier to distinguish from the unfiltered QW emission, and it has a line width of $3 \mathrm{~nm}$ around $0^{\circ}$ emission angle and close to threshold. However, the line width is likely broadened by the lateral cavity thickness variations discussed below. ${ }^{36,38}$ Nevertheless, when compared to the resolution-limited laser line widths above threshold, this confirms a clear line width narrowing.

As can be seen from Figure 2a, the VCSEL experiences multimode lasing, and the number of modes increases with higher pump power. We attribute the multimode lasing to filamentation, which is often observed in III-nitride VCSELs. ${ }^{8,22,35}$ The filamentation was investigated by spatial imaging of another device with a similar multimode spectrum but lower threshold $\left(<3 \mathrm{MW} / \mathrm{cm}^{2}\right)$, shown in Figure 3a,b. The spatial emission distribution at the sample surface shows multiple, spatially separated lasing spots. On the contrary, areas with a quasi single-mode spectrum (Figure 3c,d) show lasing confined to a single spot. The pump spot is approximately circular and Gaussian, and no correlation between the shape of the pump spot's lateral intensity profile and the spatial emission distribution from the VCSELs was observed. The substantially different emission wavelengths of separate lasing spots can be explained by the short cavity length, which will cause large spectral shifts even for small local thickness variations (see Supporting Information Section 3). For a $2.5 \lambda$ cavity, a $1 \mathrm{~nm}$ deviation in cavity length will result in a $0.5 \mathrm{~nm}$ spectral shift. Because of the thickness variations and other sample inhomogeneities, the different devices and filaments also have different lasing thresholds. Filamentation is likely promoted by the lack of intentional optical guiding structures for lateral confinement, which could be used to reduce this effect in future devices.

To further confirm lasing, the polarization of the emission was investigated. For a lasing device the polarization direction is pinned in the same direction for all individual modes for multimode lasing areas as those shown in Figure 2 and Figure $3 a, b$, but the polarization direction of the lasing emission varies across the sample. The emission from a device that showed single-mode lasing is displayed in Figure 4. The spontaneous emission around $320 \mathrm{~nm}$ only has a degree of polarization of approximately $55 \%$. In contrast, the single-mode lasing peak at $307 \mathrm{~nm}$ has a resolution-limited line width and a degree of polarization of $97 \%$.

In conclusion, we have demonstrated an optically pumped AlGaN VCSEL emitting around $310 \mathrm{~nm}$. The device was realized through substrate removal by electrochemical etching of $\mathrm{AlGaN}$, enabling the use of a top and bottom highreflectivity dielectric DBR. Lasing is confirmed by (1) a threshold in output intensity versus pump power, (2) a clear narrowing of line width and beam width at threshold, and (3) a transition to highly polarized emission above threshold. Furthermore, our method of creating vertical cavities for UVB emission is readily extendable to even shorter wavelengths, thus offering a key building block to deliver VCSELs with emission covering a major part of the UV spectral range. Together with the rapid progress in tunnel junctions for electrical injection, these results hold promise for the development of a small-footprint, power-efficient UV light source with excellent beam characteristics for medical applications and compact disinfection systems.

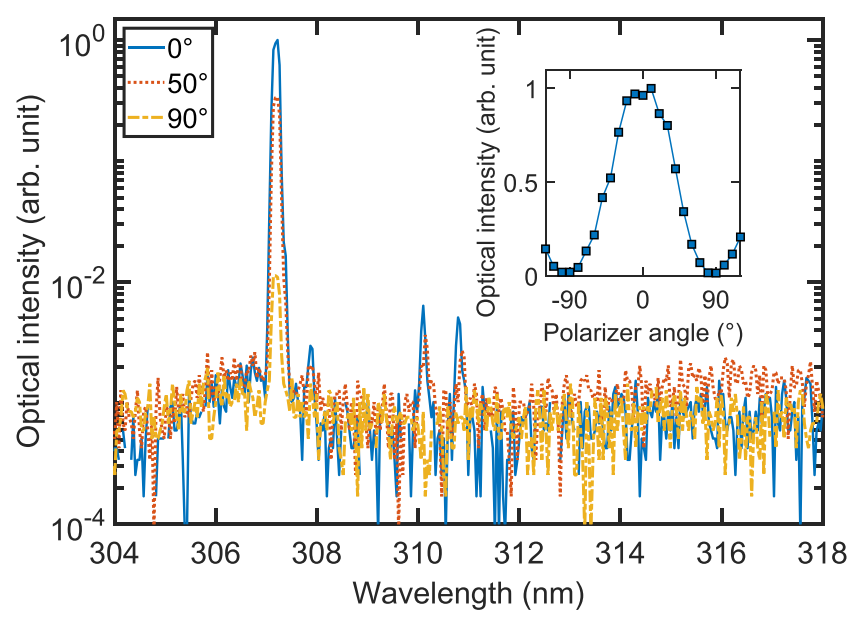

Figure 4. Polarization of UVB VCSEL emission. PL spectra of an area with single-mode emission for different polarizer angles. The inset shows the integrated optical intensity of the $307 \mathrm{~nm}$ peak as a function of the polarizer angle. The spectra were taken above threshold at $2.8 \mathrm{MW} / \mathrm{cm}^{2}$ pump power, and the obtained lasing peak has a full width at half-maximum line width of $0.15 \mathrm{~nm}$, which is the resolution limit of the setup.

\section{METHODS}

Design Simulations. A $1 \mathrm{D}$ scalar wave transfer matrix method $^{39}$ was used to calculate the reflectivity spectrum of the VCSEL DBRs (see Supporting Information Section 1). Additionally, a 1D scalar wave effective index model ${ }^{40}$ was used to compute the longitudinal optical field properties of the VCSEL (see Supporting Information Section 3).

Epitaxial Growth. The heterostructure was grown by metal-organic vapor phase epitaxy in a close-coupled showerhead reactor. TMAl, TMGa, and TEGa were used as metalorganic precursors. $\mathrm{NH}_{3}$ was used as nitrogen source, and the carrier gases were hydrogen and nitrogen. The n-type doping was realized by $\mathrm{SiH}_{4}$. Relaxed $\mathrm{Al}_{0.55} \mathrm{Ga}_{0.45} \mathrm{~N}$ :Si pseudosubstrates, similar to ref 41 , were used as template for further growth of pseudomorphically strained heterostructures (see Supporting Information Section 4). The Si-doping of this template is between $2 \times 10^{18}$ and $3 \times 10^{18} \mathrm{~cm}^{-3}$. First, a $200 \mathrm{~nm}$ thick $\mathrm{Al}_{0.50} \mathrm{Ga}_{0.50} \mathrm{~N}$ :Si layer with a reduced $\mathrm{Si}$ concentration of $0.5 \times 10^{18} \mathrm{~cm}^{-3}$ was grown in order to ensure an effective confinement of the etching process to the sacrificial layer. The sacrificial layer was composed of a $100 \mathrm{~nm}$ thick $\mathrm{Al}_{0.39} \mathrm{Ga}_{0.61}:$ Si layer with a $\mathrm{Si}$ concentration of $2 \times 10^{19}$ $\mathrm{cm}^{-3}$ and a superlattice structure consisting of five periods of $\mathrm{Al}_{0.11} \mathrm{Ga}_{0.89} \mathrm{~N}: \mathrm{Si} / \mathrm{Al}_{0.39} \mathrm{Ga}_{0.61}$ :Si layers with layer thicknesses of 5 $\mathrm{nm}$. The Si concentration was kept at around $2 \times 10^{19} \mathrm{~cm}^{-3}$. After the sacrificial layer, the lower cavity layer with a thickness of $218 \mathrm{~nm}$ and an aluminum mole fraction of $63 \%$ as measured by high resolution X-ray diffraction was grown followed by the active region. The active region starts with a $40 \mathrm{~nm}$ thick barrier, followed by three AlGaN QWs with a thickness of $2 \mathrm{~nm}$ and peak emission at $320 \mathrm{~nm}$. The target peak emission wavelength was achieved by tuning the TMAl flux, but the exact composition of the QWs is unknown. The QWs are separated by $5 \mathrm{~nm}$ thick $\mathrm{Al}_{0.50} \mathrm{Ga}_{0.50} \mathrm{~N}$ barriers. The active region is capped by a $15 \mathrm{~nm}$ thick last $\mathrm{Al}_{0.50} \mathrm{Ga}_{0.50} \mathrm{~N}$ barrier and finally an upper cavity layer with a thickness of $42 \mathrm{~nm}$ and the same aluminum mole fraction as the lower cavity layer. No $\mathrm{SiH}_{4}$ was supplied to the reactor chamber during the VCSEL structure growth to prevent any parasitic etching during the 
electrochemical etching of the sacrificial layer. A transmission electron microscopy study of a similar epitaxial VCSEL structure is presented in Supporting Information Section 5.

Device Fabrication. The device fabrication, schematically displayed in Supporting Information Figure S7, started with an initial mesa dry etch using $\mathrm{Ar} / \mathrm{Cl}_{2}$ chemistry into the current spreading layer to expose the sacrificial layer. Then, on a separate part of the chip, a 20/80/20/100 nm Ti/Al/Ti/Au contact used for the bias voltage during electrochemical etching was evaporated and annealed for $1 \mathrm{~min}$ at $900{ }^{\circ} \mathrm{C}$. After the annealing, an 11-pair $\mathrm{HfO}_{2} / \mathrm{SiO}_{2}$ DBR was deposited by reactive radio frequency (RF) magnetron sputtering using an FHR MS150 system having $200 \mathrm{~mm}$ metal targets. Deposition conditions were $0.75 \mathrm{~kW}$ RF power, $80 \mathrm{sccm}$ Ar flow, $6 \mathrm{sccm} \mathrm{O}$ flow, and $1.4 \times 10^{-2}$ mbar pressure for $\mathrm{HfO}_{2}$ and $1.0 \mathrm{~kW}$ RF power, $40 \mathrm{sccm}$ Ar flow, $15 \mathrm{sccm} \mathrm{\textrm {O } _ { 2 }}$ flow, and $1.3 \times 10^{-2}$ mbar pressure for $\mathrm{SiO}_{2}$. The thicknesses were $36.2 \mathrm{~nm}$ for $\mathrm{HfO}_{2}$ and $53.8 \mathrm{~nm}$ for $\mathrm{SiO}_{2}$, corresponding to a stop band center at $320 \mathrm{~nm}$, except for the final $\mathrm{HfO}_{2}$ layer, which was $60.6 \mathrm{~nm}$ thick to adjust for the phase shift after reflection on the $\mathrm{Al}$ mirror. Subsequently, $\mathrm{NF}_{3}$ dry etching of the DBR exposed the contact and one edge of each mesa. A 50 $\mathrm{nm}$ thick $\mathrm{Al}$ mirror was then evaporated on the DBRs on the mesas, followed by a $20 \mathrm{~nm}$ sputtered $\mathrm{SiO}_{2}$ layer to prevent alloying between the $\mathrm{Al}$ layer and the subsequently evaporated $10 / 200 \mathrm{~nm} \mathrm{Ti} / \mathrm{Au}$ pad for bonding. As a last step before electrochemical etching, the whole sample surface was protected by photoresist except the electrochemical etch contact and the openings in the DBR at each mesa.

The electrochemical etching of the sacrificial layer was carried out in a two-electrode setup using $0.3 \mathrm{M}$ nitric acid as the electrolyte. A constant positive potential of $30 \mathrm{~V}$ was applied to the sample relative to a graphite counter electrode, leading to anodic etching. The etching was done at room temperature with no intentional illumination, and the electrolyte was stirred using a magnetic stir bar. The progress of the etching was monitored in situ using a stereomicroscope, and the etching of the mesas was completed after $35 \mathrm{~min}$. After electrochemical etching, the resist was removed, and the mesas were transferred to a $10 / 300 \mathrm{~nm} \mathrm{Ti} / \mathrm{Au}$ covered $\mathrm{Si}$ chip by thermocompression bonding in a vacuum for $2 \mathrm{~h}$ at $300{ }^{\circ} \mathrm{C}$ with a pressure of approximately $50 \mathrm{MPa}$. Top-view and crosssectional scanning electron microscope (SEM) images of a mesa after bonding are shown in Figure S8. Finally, a 10-pair $\mathrm{HfO}_{2} / \mathrm{SiO}_{2}$ DBR was sputtered on top using the same depositions conditions as for the first DBR. Using a Agilent Cary 5000 spectrophotometer with a VW specular reflectance accessory, the 10-pair DBR on a reference $\mathrm{Si}$ wafer was measured to have a peak reflectivity over 99\% (see Supporting Information Figure S1). Additionally, for the VCSEL sample, a $10.4 \mathrm{~nm} \mathrm{HfO}_{2}$ layer was sputtered before the final DBR to partly compensate for the epitaxially grown AlGaN device layers being thinner than targeted. In total, several 10s of mesas with areas ranging from 5000 to $90000 \mu \mathrm{m}^{2}$ were successfully fabricated.

Photoluminescence Measurements. For optical pumping (see setup in Supporting Information Figure S9), a CryLaS FQSS 266-200 frequency quadrupled Nd:YAG laser $(266 \mathrm{~nm}$ ) with a repetition rate of $60 \mathrm{~Hz}$ and a pulse duration of $1.3 \mathrm{~ns}$ was used together with neutral density filters to control the pumping power. Low-frequency and long pulse duration were chosen in order to provide quasi-continuous pump conditions with respect to the lifetimes of the excited states but preventing extensive heating of the sample. The diameter of the excitation spot was estimated to be $10-15 \mu \mathrm{m}$, and for the power density calculations a diameter of $12 \mu \mathrm{m}$ was used. The measurements were performed at room temperature and on at least 100 different positions, out of which almost all lased. Uninterrupted pumping during more than $1 \mathrm{~h}$ slightly over threshold (a few $\mathrm{MW} / \mathrm{cm}^{2}$ ) did not lead to any reduction in emission intensity. A direct "one-shot" visualization of the angle-dependent farfield emission was realized by using a microscope objective with $\mathrm{NA}=0.4$ for collecting the angular distribution between $\pm 23.6^{\circ}$ and two lenses in the $4 \mathrm{f}$-arrangement to image the Fourier plane on the spectrometer slit, where the angular information was wavelength resolved and imaged on a Peltiercooled CCD chip with $1024 \times 256$ pixels. The result is a 3D graph showing the emission intensity versus wavelength and angle (equivalent to energy and momentum). The data in Figure $2 \mathrm{a}, \mathrm{b}$ were measured by integration over all collected emission angles, and the optical intensity, $I$, in the inset of Figure $2 \mathrm{~b}$ was fitted to $I \propto r-1+\sqrt{(r-1)^{2}+4 \beta r}$, where $r$ is the pump power normalized to the threshold pump power. The dashed line in Figure $2 \mathrm{~d}$ marking the longitudinal cavity mode was simulated using the transfer matrix method assuming an AlGaN cavity with a thickness $25 \mathrm{~nm}$ shorter than designed and accounting for the $10.4 \mathrm{~nm} \mathrm{HfO}_{2}$ layer before the top DBR. Furthermore, the spatial emission distribution at the sample surface was captured using a beam profiling camera, and the degree of polarization was measured by adding a polarizer to the collection beam path and varying the polarizer angle. The degree of polarization was calculated as $\left(I_{\max }-I_{\min }\right) /\left(I_{\max }+I_{\min }\right)$, where $I_{\max }$ is the maximum and $I_{\min }$ the minimum optical intensity.

\section{ASSOCIATED CONTENT}

\section{Supporting Information}

The Supporting Information is available free of charge at https://pubs.acs.org/doi/10.1021/acsphotonics.0c01382.

Details on DBR reflectivity, threshold carrier density, VCSEL design and resonance wavelength, strain state, structural analysis, process flow, and photoluminescence measurements (PDF)

\section{AUTHOR INFORMATION}

\section{Corresponding Author}

Filip Hjort - Department of Microtechnology and Nanoscience, Chalmers University of Technology, 41296 Gothenburg, Sweden; (1) orcid.org/0000-0003-3694-3644; Email: filip.hjort@chalmers.se

\section{Authors}

Johannes Enslin - Institute of Solid State Physics, Technische Universität Berlin, 10623 Berlin, Germany

Munise Cobet - Institute of Solid State Physics, Technische Universität Berlin, 10623 Berlin, Germany

Michael A. Bergmann - Department of Microtechnology and Nanoscience, Chalmers University of Technology, 41296 Gothenburg, Sweden; 10 orcid.org/0000-0001-6885-799X

Johan Gustavsson - Department of Microtechnology and Nanoscience, Chalmers University of Technology, 41296 Gothenburg, Sweden

Tim Kolbe - Ferdinand-Braun-Institut, Leibniz-Institut für Höchstfrequenztechnik, 12489 Berlin, Germany 
Arne Knauer - Ferdinand-Braun-Institut, Leibniz-Institut für Höchstfrequenztechnik, 12489 Berlin, Germany

Felix Nippert - Institute of Solid State Physics, Technische Universität Berlin, 10623 Berlin, Germany

Ines Häusler - Institute of Optics and Atomic Physics, Technische Universität Berlin, 10623 Berlin, Germany

Markus R. Wagner - Institute of Solid State Physics, Technische Universität Berlin, 10623 Berlin, Germany; (1) orcid.org/0000-0002-7367-5629

Tim Wernicke - Institute of Solid State Physics, Technische Universität Berlin, 10623 Berlin, Germany

Michael Kneissl - Institute of Solid State Physics, Technische Universität Berlin, 10623 Berlin, Germany

Åsa Haglund - Department of Microtechnology and Nanoscience, Chalmers University of Technology, 41296 Gothenburg, Sweden

Complete contact information is available at: https://pubs.acs.org/10.1021/acsphotonics.0c01382

\section{Author Contributions}

Å.H., M.K., and T.W. proposed and supervised the project. J.G. performed the VCSEL design simulations. T.K. and A.K. developed and grew the pseudosubstrate. J.E. grew the rest of the epitaxial heterostructures, performed the X-ray diffraction analysis, and together with F.H. conducted the atomic force microscopy measurements. F.H. developed the fabrication process and performed the device processing with assistance from J.E. and M.A.B. M.A.B. built the setup for electrochemical etching and developed the etching process. M.C. built the PL measurement setup and did the majority of the PL measurement. F.H. conducted part of the PL measurements and performed the data analysis together with M.C. I.H. performed the transmission electron microscopy measurements. F.N. performed the microphotoluminescence measurements under supervision of M.R.W. F.H. wrote the manuscript with input from the other coauthors.

\section{Notes}

The authors declare no competing financial interest.

\section{ACKNOWLEDGMENTS}

The authors thank Sylvia Hagedorn from Ferdinand-BraunInstitute for growing the AlN template and Michael Winkler, Martin Feneberg, and Rüdiger Goldhahn from Otto-vonGuericke-Universität Magdeburg for supplying dielectric functions of AlGaN. This work was performed in part at Myfab Chalmers, and the project was financially supported by the Swedish Research Council (2018-00295), the Swedish Foundation for Strategic Research (IB13-0004), the European Research Council (ERC) under the European Union's Horizon 2020 research and innovation program (grant agreement no. 865622), the German Federal Ministry of Education and Research (BMBF) within the "Advanced UV for Life" project, and the Deutsche Forschungsgemeinschaft (DFG) within the Collaborative Research Center "Semiconductor Nanophotonics" (SFB 787). The TEM images were carried out as part of the DFG core facility project Berlin Electron Microscopy Network (Berlin EM Network).

\section{REFERENCES}

(1) Michalzik, R. VCSELs: Fundamentals, Technology and Applications of Vertical-Cavity Surface-Emitting Lasers; Springer Berlin Heidelberg: Berlin, Heidelberg, 2013; Vol. 166; pp 19-75.
(2) Moench, H.; Conrads, R.; Deppe, C.; Derra, G.; Gronenborn, S.; Gu, X.; Heusler, G.; Kolb, J.; Miller, M.; Pekarski, P.; PollmannRetsch, J.; Pruijmboom, A.; Weichmann, U. High-power VCSEL systems and applications. Proc. SPIE 2015, 9348, 93480W.

(3) Kuramoto, M.; Kobayashi, S.; Akagi, T.; Tazawa, K.; Tanaka, K.; Saito, T.; Takeuchi, T. High-output-power and high-temperature operation of blue GaN-based vertical-cavity surface-emitting laser. Appl. Phys. Express 2018, 11, 112101.

(4) Kuramoto, M.; Kobayashi, S.; Akagi, T.; Tazawa, K.; Tanaka, K.; Nakata, K.; Saito, T. Watt-class blue vertical-cavity surface-emitting laser arrays. Appl. Phys. Express 2019, 12, No. 091004.

(5) Hamaguchi, T.; Nakajima, H.; Tanaka, M.; Ito, M.; Ohara, M.; Jyoukawa, T.; Kobayashi, N.; Matou, T.; Hayashi, K.; Watanabe, H.; Koda, R.; Yanashima, K. Submilliampere-threshold continuous wave operation of GaN-based vertical-cavity surface-emitting laser with lateral optical confinement by curved mirror. Appl. Phys. Express 2019, 12, No. 044004.

(6) Mei, Y.; Weng, G.-E.; Zhang, B.-P.; Liu, J.-P.; Hofmann, W.; Ying, L.-Y.; Zhang, J.-Y.; Li, Z.-C.; Yang, H.; Kuo, H.-C. Quantum dot vertical-cavity surface-emitting lasers covering the 'green gap'. Light: Sci. Appl. 2017, 6, No. e16199.

(7) Lee, S.; Forman, C. A.; Kearns, J.; Leonard, J. T.; Cohen, D. A.; Nakamura, S.; DenBaars, S. P. Demonstration of GaN-based verticalcavity surface-emitting lasers with buried tunnel junction contacts. Opt. Express 2019, 27, 31621-31628.

(8) Mishkat-Ul-Masabih, S. M.; Aragon, A. A.; Monavarian, M.; Luk, T. S.; Feezell, D. F. Electrically injected nonpolar GaN-based VCSELs with lattice-matched nanoporous distributed Bragg reflector mirrors. Appl. Phys. Express 2019, 12, No. 036504.

(9) Chang, T.-C.; Kuo, S.-Y.; Lian, J.-T.; Hong, K.-B.; Wang, S.-C.; $\mathrm{Lu}, \mathrm{T}$.-C. High-temperature operation of GaN-based vertical-cavity surface-emitting lasers. Appl. Phys. Express 2017, 10, 112101.

(10) Takeuchi, T.; Kamiyama, S.; Iwaya, M.; Akasaki, I. GaN-based vertical-cavity surface-emitting lasers with AlInN/GaN distributed Bragg reflectors. Rep. Prog. Phys. 2019, 82, No. 012502.

(11) Hashemi, E.; Bengtsson, J.; Gustavsson, J.; Stattin, M.; Cosendey, G.; Grandjean, N.; Haglund, Å. Analysis of structurally sensitive loss in GaN-based VCSEL cavities and its effect on modal discrimination. Opt. Express 2014, 22, 411-426.

(12) Zhang, C.; ElAfandy, R.; Han, J. Distributed Bragg Reflectors for GaN-Based Vertical-Cavity Surface-Emitting Lasers. Appl. Sci. 2019, 9, 1593.

(13) Leonard, J. T.; Cohen, D. A.; Yonkee, B. P.; Farrell, R. M.; DenBaars, S. P.; Speck, J. S.; Nakamura, S. Smooth e-beam-deposited tin-doped indium oxide for III-nitride vertical-cavity surface-emitting laser intracavity contacts. J. Appl. Phys. 2015, 118, 145304.

(14) Kneissl, M.; Seong, T.-Y.; Han, J.; Amano, H. The emergence and prospects of deep-ultraviolet light-emitting diode technologies. Nat. Photonics 2019, 13, 233-244.

(15) Redwing, J. M.; Loeber, D. A. S.; Anderson, N. G.; Tischler, M. A.; Flynn, J. S. An optically pumped $\mathrm{GaN}-\mathrm{AlGaN}$ vertical cavity surface emitting laser. Appl. Phys. Lett. 1996, 69, 1-3.

(16) Someya, T.; Werner, R.; Forchel, A.; Catalano, M.; Cingolani, R.; Arakawa, Y. Room Temperature Lasing at Blue Wavelengths in Gallium Nitride Microcavities. Science 1999, 285, 1905-1906.

(17) Zhou, H.; Diagne, M.; Makarona, E.; Nurmikko, A. V.; Han, J.; Waldrip, K. E.; Figiel, J. J. Near ultraviolet optically pumped vertical cavity laser. Electron. Lett. 2000, 36, 1777-1779.

(18) Onishi, T.; Imafuji, O.; Nagamatsu, K.; Kawaguchi, M.; Yamanaka, K.; Takigawa, S. Continuous Wave Operation of GaN Vertical Cavity Surface Emitting Lasers at Room Temperature. IEEE J. Quantum Electron. 2012, 48, 1107-1112.

(19) Chen, R.; Sun, H. D.; Wang, T.; Hui, K. N.; Choi, H. W. Optically pumped ultraviolet lasing from nitride nanopillars at room temperature. Appl. Phys. Lett. 2010, 96, 241101.

(20) Liu, Y.-S.; Saniul Haq, A. F. M.; Mehta, K.; Kao, T.-T.; Wang, S.; Xie, H.; Shen, S.-C.; Yoder, P. D.; Ponce, F. A.; Detchprohm, T.; Dupuis, R. D. Optically pumped vertical-cavity surface-emitting laser 
at $374.9 \mathrm{~nm}$ with an electrically conducting n-type distributed Bragg reflector. Appl. Phys. Express 2016, 9, 111002.

(21) Zhang, C.; El Afandy, R. T.; Zhang, J.; Chen, S.; Nurmikko, A.; Han, J. Development of nanopore-based near ultraviolet verticalcavity surface emitting lasers. Proc. SPIE 2019, 10918, 109181M.

(22) Leonard, J. T.; Cohen, D. A.; Yonkee, B. P.; Farrell, R. M.; Margalith, T.; Lee, S.; DenBaars, S. P.; Speck, J. S.; Nakamura, S. Nonpolar III-nitride vertical-cavity surface-emitting lasers incorporating an ion implanted aperture. Appl. Phys. Lett. 2015, 107, No. 011102.

(23) Zhang, Y.; Jamal-Eddine, Z.; Akyol, F.; Bajaj, S.; Johnson, J. M.; Calderon, G.; Allerman, A. A.; Moseley, M. W.; Armstrong, A. M.; Hwang, J.; Rajan, S. Tunnel-injected sub $290 \mathrm{~nm}$ ultra-violet light emitting diodes with $2.8 \%$ external quantum efficiency. Appl. Phys. Lett. 2018, 112, No. 071107.

(24) Kuhn, C.; Sulmoni, L.; Guttmann, M.; Glaab, J.; Susilo, N.; Wernicke, T.; Weyers, M.; Kneissl, M. MOVPE-grown AlGaN-based tunnel heterojunctions enabling fully transparent UVC LEDs. Photonics Res. 2019, 7, B7-B11.

(25) Clinton, E. A.; Engel, Z.; Vadiee, E.; Carpenter, J. V.; Holman, Z. C.; Doolittle, W. A. Ultra-wide-bandgap AlGaN homojunction tunnel diodes with negative differential resistance. Appl. Phys. Lett. 2019, 115, No. 082104.

(26) Brummer, G.; Nothern, D.; Nikiforov, A. Y.; Moustakas, T. D. Deep ultraviolet distributed Bragg reflectors based on graded composition AlGaN alloys. Appl. Phys. Lett. 2015, 106, 221107.

(27) Detchprohm, T.; Liu, Y.-S.; Mehta, K.; Wang, S.; Xie, H.; Kao, T.-T.; Shen, S.-C.; Yoder, P. D.; Ponce, F. A.; Dupuis, R. D. Sub $250 \mathrm{~nm}$ deep-UV AlGaN/AlN distributed Bragg reflectors. Appl. Phys. Lett. 2017, 110, No. 011105.

(28) Franke, A.; Hoffmann, M. P.; Kirste, R.; Bobea, M.; Tweedie, J.; Kaess, F.; Gerhold, M.; Collazo, R.; Sitar, Z. High reflectivity IIInitride UV-C distributed Bragg reflectors for vertical cavity emitting lasers. J. Appl. Phys. 2016, 120, 135703.

(29) Zheng, Z.; Li, Y.; Paul, O.; Long, H.; Matta, S.; Leroux, M.; Brault, J.; Ying, L.; Zheng, Z.; Zhang, B. Loss analysis in nitride deep ultraviolet planar cavity. J. Nanophotonics 2018, 12, No. 043504.

(30) Cho, H. K.; Krüger, O.; Külberg, A.; Rass, J.; Zeimer, U.; Kolbe, T.; Knauer, A.; Einfeldt, S.; Weyers, M.; Kneissl, M. Chip design for thin-film deep ultraviolet LEDs fabricated by laser lift-off of the sapphire substrate. Semicond. Sci. Technol. 2017, 32, 12LT01.

(31) Zheng, Z.; Long, H.; Matta, S.; Leroux, M.; Brault, J.; Ying, L.; Zheng, Z.; Zhang, B. Photoassisted chemical smoothing of AlGaN surface after laser lift-off. J. Vac. Sci. Technol., B: Nanotechnol. Microelectron.: Mater., Process., Meas., Phenom. 2020, 38, No. 042207.

(32) Bergmann, M. A.; Enslin, J.; Yapparov, R.; Hjort, F.; Wickman, B.; Marcinkevičius, S.; Wernicke, T.; Kneissl, M.; Haglund, Å. Electrochemical etching of $\mathrm{AlGaN}$ for the realization of thin-film devices. Appl. Phys. Lett. 2019, 115, 182103.

(33) Bergmann, M. A.; Enslin, J.; Hjort, F.; Wernicke, T.; Kneissl, M.; Haglund, Å. Thin-film flip-chip UVB LEDs realized by electrochemical etching. Appl. Phys. Lett. 2020, 116, 121101.

(34) Mehta, K.; Detchprohm, T.; Park, Y. J.; Liu, Y.-S.; Moreno, O.; Alugubelli, S. R.; Wang, S.; Ponce, F. A.; Shen, S.-C.; Dupuis, R. D.; Yoder, P. D. High Reflectivity Hybrid AlGaN/Silver Distributed Bragg Reflectors for Use in the UV-Visible Spectrum. IEEE J. Quantum Electron. 2017, 53, 1-8.

(35) Wang, S.-C.; Lu, T.-C.; Kao, C.-C.; Chu, J.-T.; Huang, G.-S.; Kuo, H.-C.; Chen, S.-W.; Kao, T.-T.; Chen, J.-R.; Lin, L.-F. Optically Pumped GaN-based Vertical Cavity Surface Emitting Lasers: Technology and Characteristics. Jpn. J. Appl. Phys. 2007, 46, 53975407.

(36) Feltin, E.; Christmann, G.; Dorsaz, J.; Castiglia, A.; Carlin, J.-F.; Butté, R.; Grandjean, N.; Christopoulos, S.; Baldassarri Höger von Högerstahl, G.; Grundy, A. J. D.; Lagoudakis, P. G.; Baumberg, J. J. Blue lasing at room temperature in an optically pumped latticematched AlInN/GaN VCSEL structure. Electron. Lett. 2007, 43, 924926.
(37) Björk, G.; Heitmann, H.; Yamamoto, Y. Spontaneous-emission coupling factor and mode characteristics of planar dielectric microcavity lasers. Phys. Rev. A: At., Mol., Opt. Phys. 1993, 47, $4451-4463$.

(38) Christmann, G.; Simeonov, D.; Butté, R.; Feltin, E.; Carlin, J. F.; Grandjean, N. Impact of disorder on high quality factor III-V nitride microcavities. Appl. Phys. Lett. 2006, 89, No. 261101.

(39) Saleh, B. E. A.; Teich, M. C. Fundamentals of Photonics, 2nd ed.; John Wiley \& Sons, Inc.: Hoboken, NJ, USA, 2007; pp 243-279.

(40) Hadley, G. R. Effective index model for vertical-cavity surfaceemitting lasers. Opt. Lett. 1995, 20, 1483-1485.

(41) Enslin, J.; Mehnke, F.; Mogilatenko, A.; Bellmann, K.; Guttmann, M.; Kuhn, C.; Rass, J.; Lobo-Ploch, N.; Wernicke, T.; Weyers, M.; Kneissl, M. Metamorphic $\mathrm{Al}_{0.5} \mathrm{Ga}_{0.5} \mathrm{~N}: \mathrm{Si}$ on AlN/sapphire for the growth of UVB LEDs. J. Cryst. Growth 2017, 464, 185-189. 